\title{
Patients' preferences for involvement in treatment decision making
} in Japan

\author{
Miho Sekimoto*1, Atsushi Asai², Motoki Ohnishi ${ }^{3}$, Etsuyo Nishigaki ${ }^{4}$, \\ Tsuguya Fukui ${ }^{3}$, Takuro Shimbo ${ }^{3}$ and Yuichi Imanaka ${ }^{1}$
}

\begin{abstract}
Address: ${ }^{1}$ Department of Healthcare Economics and Quality Management, Kyoto University Graduate School of Medicine, Kyoto, Japan, 2Department of Bioethics, Kyoto University Graduate School of Medicine, Kyoto, Japan, ${ }^{3}$ Department of General Medicine and Clinical Epidemiology, Kyoto University Graduate School of Medicine, Kyoto, Japan and ${ }^{4}$ Department of Social Psychology, Wakayama Medical College, Wakayama, Japan

Email: Miho Sekimoto* - mihoseki@kuhp.kyoto-u.ac.jp; Atsushi Asai - aasai@pbh.med.kyoto-u.ac.jp; Motoki Ohnishi - mot@kuhp.kyotou.ac.jp; Etsuyo Nishigaki - etsuyo@wakayama-med.ac.jp; Tsuguya Fukui - fkts@kuhp.kyoto-u.ac.jp; Takuro Shimbo - shimbot@kuhp.kyotou.ac.jp; Yuichi Imanaka - imanaka@pbh.med.kyoto-u.ac.jp

* Corresponding author
\end{abstract}

Published: 0 I March 2004

BMC Family Practice 2004, 5:I

This article is available from: http://www.biomedcentral.com/l47/-2296/5/I media for any purpose, provided this notice is preserved along with the article's original URL.

Received: 0 I December 2003

Accepted: 0I March 2004

\begin{abstract}
Background: A number of previous studies have suggested that the Japanese have few opportunities to participate in medical decision-making, as a result both of entrenched physician paternalism and national characteristics of dependency and passivity. The hypothesis that Japanese patients would wish to participate in treatment decision-making if adequate information were provided, and the decision to be made was clearly identified, was tested by interview survey.

Methods: The subjects were diabetic patients at a single outpatient clinic in Kyoto. One of three case study vignettes (pneumonia, gangrene or cancer) was randomly assigned to each subject and, employing face-to-face interviews, the subjects were asked what their wishes would be as patients, for treatment information, participation in decision-making and family involvement.

Results: 134 patients participated in the study, representing a response rate of $90 \%$. The overall proportions of respondents who preferred active, collaborative, and passive roles were $12 \%$, $71 \%$, and $17 \%$, respectively. Respondents to the cancer vignette were less likely to prefer an active role and were more likely to prefer family involvement in decision-making compared to non-cancer vignette respondents. If a physician's recommendation conflicted with their own wishes, $60 \%$ of the respondents for each vignette answered that they would choose to respect the physician's opinion, while few respondents would give the family's preference primary importance.

Conclusions: Our study suggested that a majority of Japanese patients have positive attitudes towards participation in medical decision making if they are fully informed. Physicians will give greater patient satisfaction if they respond to the desire of patients for participation in decisionmaking.
\end{abstract}

\section{Background}

The current dominant ideology of health care in Western countries supports the active participation of patients in decision-making, but this is less accepted in Japan where 
physician paternalism remains dominant [1]. Recent studies conducted in Japan have produced contradictory results as regards patients' desire for information and for self-determination. Kubo and Ishizaki reported that a majority of Japanese patients thought the physician should make the final decision [2,3], while Asai and Toyokawa found Japanese patients were dissatisfied with the current situation and wanted to actively participate in decision-making regarding their treatment [4-6].

Medical choices include two elements: problem-solving (PS) and decision-making (DM) [7]. PS involves identifying the single or most correct solution to a problem, which usually requires expertise, and in which patient involvement can only have a limited role. By contrast, DM involves making a choice, often involving trade-offs, from a number of possible alternatives. Such processes as making diagnoses and identifying treatment options, risks, and benefits, and the outcome probabilities associated with each option, belong to PS tasks, while those of determining overall utilities and tailoring to patient idiosyncracies belong to DM tasks [7-9].

Although DM and PS tasks are not always mutually exclusive, the distinction between them is important in the context of understanding the expectations and limits of patient autonomy. When patients are presented with PS tasks, they are usually happy to hand these over to "experts". However, this response does not mean that such patients do not, nevertheless, wish to participate in decision making when the PS tasks are completed and provided decision points for care or treatment are clearly identified. Some previous studies in Japan, which have sought to ascertain patients' desire for autonomy [2,3], have failed to distinguish between PS and DM tasks. As a result, the patients' desire for participation in decision making may have been underestimated. However, because there is no room for PS tasks in cases of terminal illness, similar studies on advanced directives may possibly have successfully revealed the level of patients' desire for autonomy [5]. However, no study of patients' desire for participation in decision making that explicitly discriminates between PS and DM tasks has yet been conducted in Japan.

True self-determination cannot be achieved without knowledge of the disease and its treatment options. Japanese patients have limited opportunities for self-determination because the information given them is unsatisfactory from several points of view. Firstly, Japanese physicians tend to think that patients in general will not understand and that therefore it is futile, or worse, to give difficult medical information; they have expressed concern that imparting complicated medical information is likely to result in unnecessary anxiety and confusion
[10]. Secondly, it is a common practice among Japanese physicians to withhold 'bad news' from patients and, especially in the case of cancer, to inform family members first, in the belief that this is the kindest way to proceed [11-14]. The diagnosis is revealed to the family, who then make the decision as to whether or not to inform the patient. Many patients do not like to ask for withheld information, even when they want to be told the truth [12].

In countries such as Japan, where the patient's family is often informed first, and therefore plays an important role in decision making, the degree of patient involvement in medical decision making is very much controlled by family members. Some physicians consider the family-physician relationship more important than the physicianpatient relationship [14]. Ruhnke et al. surveyed the attitudes of Japanese physicians and patients to ethical decision making and patient autonomy, and found that $80 \%$ of physicians and $65 \%$ of patients agreed that the doctor should report the diagnosis of incurable cancer to a patient's family first, and should also let them decide whether or not the patient was to be informed [15]. In the same survey, only $24 \%$ of Japanese patients agreed with disclosure of a diagnosis of incurable cancer to a patient, if the patient's family did not want the patient to be told.

Existing models for patient autonomy [16-19] envision a dyadic interaction between patient and physician, and so may not be suitable for the situation in Japan. It would be interesting to know to what extent the family-patient relationship in Japan is conflictual or supportive, and how it affects the clinical relationship. As a first step, the preferred roles of Japanese patients in medical decision-making, vis-a-vis both physicians and family members, need to be clarified.

This study was planned to investigate the following questions:

1. Would patients want to participate in treatment decision making if complete information regarding their diagnosis and treatment options were given, and if the decisions to be made were clearly identified?

2. What are the factors (e.g. patients' characteristics, severity of disease) that most affect patients' desire to participate in DM?

3. What roles do patients want physicians and family members to play in treatment decisions? 


\section{Methods \\ Subjects}

The subjects were patients with type 2 diabetes mellitus attending Kyoto Preventative Medical Center, a consulting clinic attached to a health-screening center foundation in Kyoto City. With their doctors' permission, we recruited a sample from among patients cared for by two physicians and who attended the clinic between 14 February, 2000 and 6 May, 2000. The study took the form of an interview survey. Patients seeking treatment at the clinic on the days the interviewer was present were randomly approached by one of the authors (MS). The purpose of the study was explained and they were asked to participate. Patients were also told that they had the right to refuse to participate in any portion of the interview and that their refusal would not cause them disadvantages. This was all expressed to eligible patients in written form. Patients who agreed were interviewed face-to-face and the subjects' responses were tape-recorded.

We did not seek Institutional Review Board (IRB)'s approval for this study for the following reasons. In 2000, when this study was designed, Japan's IRBs did not demand ethical review for research involving interview surveys, and the Ethics Review Committee of Kyoto University, at that time, reviewed only research protocols for clinical trials. Given these circumstances, we opted for an unofficial discussion on the ethical issues pertaining to this study. Members of this discussion included faculty in the Department of General Medicine and Clinical Epidemiology, Kyoto University. This discussion concluded that inherent risks could be controlled by ensuring participants' voluntariness and data confidentiality. The research protocol was proposed to Kyoto Preventative Medical Center where this study was conducted and was approved by the institution.

\section{Questionnaire contents}

The questionnaire details are shown in the appendix. Original vignettes were written in Japanese and all interviews were conducted in Japanese. Prior to the interview, the subjects were given a brief outline of the study and were assured of the confidentiality of their responses. The introductory section of the questionnaire covered sociodemographic information, including age, sex, marital status, employment status, family members, and years of schooling.

The three vignettes, presenting pneumonia, gangrene and cancer, represented different levels of severity of illness and difficulty in decision-making. In these terms, pneumonia is mild disease; gangrene a severe and high risk disease, but with benefits of treatment which are relatively easy to understand; and cancer a severe life-threatening disease. One of the vignettes was randomly allocated to each sub- ject, using lots. First, the allocated vignette was presented to the subject, who was then asked by the interviewer whether he/she would consult a physician (Question A). After the diagnosis for each vignette was revealed, the subject's knowledge of treatment options was explored (Question B). The interviewer then provided information about diagnosis, treatment options, and prognosis, including risks, benefits and outcome probability, so that the subject without prior knowledge or PS capacity could participate in associated decision making. The subject was then asked questions regarding his/her anticipated desire for treatment information, personal DM participation, family involvement in decision making, and knowledge of the probability of various treatment outcomes (from $\mathrm{C}$ through $\mathrm{G}$ in the appendix).

\section{Statistical analysis}

Normally distributed variables were compared using Student's t-test and one-way ANOVA. Responses to questions on the patient's desire for medical information (Question C-1 through C-6) were coded on a scale of 1 to 3 , with 1 representing not at all; 2, partially; and 3, fully. Ordinal variables were compared using the Kruscal-Wallis test. The patients' preferred roles in decision making were classified into three categories using similar criteria to those used in previous studies $[7,20]$, as follows: "active" if the answer to Question E ('Who should decide which treatment option should be selected?') was "myself alone" or "myself and my family alone"; "passive" if the answer was "the doctor and my family alone" or "the doctor alone"; and "collaborative" if the answer was "myself and the doctor alone" or "the doctor, myself and my family equally". Patient desire for family involvement was classified into two categories: "wish" if the answer to Question E was "the doctor and my family alone" or "myself and my family alone" or "the doctor, myself and my family equally"; and "do not wish" if the answer was "myself alone" or "the doctor alone" or "myself and the doctor alone". Nominal variables were analyzed using the chi-square test or Fisher's exact test. ANCOVA was performed to identify variables associated with the patient's preferred role in decision making and the patient's preference for family involvement in decision-making. All analyses were performed with STATA 7.0 (Stata Cooperation, Collage Station, Texas, USA).

\section{Results \\ Characteristics of respondents}

Of 149 patients who were approached, 134 (90\%) agreed to participate in the study. One patient refused to continue in the middle of the interview. There was no statistical difference in age $(\mathrm{P}=0.88)$ and sex distribution $(\mathrm{P}=$ 0.75 ) between the participants and non-participants. Key background information is summarized in Table 1 . There were twice as many male $(n=94)$ as female $(n=39)$ 
respondents, reflecting the gender distribution of outpatients at the clinic. The mean age of male respondents was lower than that of female respondents $(56.8 \pm 11.3$ vs. $60.9 \pm 10.0, \mathrm{P}=0.06)$. The proportion who were employed was significantly higher in male than in female respondents ( $84 \%$ vs. $46 \%, \mathrm{P}=0.0001)$, as was the mean years of schooling $(14.0 \pm 2.7$ vs. $12.4 \pm 2.4, \mathrm{P}=0.001)$. Most of the respondents (93\%) lived with other family members. The numbers of respondents who responded to the pneumonia, gangrene and cancer vignette were 52, 37 and 44 , respectively. No difference was observed in the distribution of age $(P=0.56)$, sex $(P=0.63)$, marital status $(P=0.21)$ and employment status $(P=0.15)$ in the three vignette subject groups.

\section{Knowledge of treatment options and desire for information}

Table 2 shows the mean scores and percentages for each questionnaire item (except A) for each of the three vignettes. All respondents but one in the pneumonia vignette group replied to Question A that they would consult a doctor. More than $90 \%$ of respondents for each vignette said they had little or no knowledge of the treatment options, and there was no statistically significant difference between the three vignettes as regards expressed desire for information, except as regards the wish to be given the name of a famous specialist in the disease area concerned, which was significantly higher in the cancer vignette group.

All respondents except one expressed a preference to be informed directly by a doctor, and about two thirds would wish to share the information with their family members. Respondents who were allocated the cancer vignette were more likely to indicate a wish to share the information with their families than were those allocated the pneumonia vignette ( $82 \%$ vs. $52 \%, \mathrm{P}=0.003)$.

\section{Preference for participation in medical decision making}

A majority of the respondents for each vignette $(68 \%$ to $75 \%$ ) expressed a preference for making treatment decisions in collaboration with a physician. Only 16\% (22 of 133 respondents) expressed a preference for delegating decision-making to their families or to the physician alone. The proportion of patients who expressed a preference for decision-making without involving the physician was small - $16 \%$ for each of the pneumonia and gangrene vignettes, and $7 \%$ for the cancer vignette. Considerably more expressed a preference for involving their families [ $42 \%$ for the pneumonia vignette, $41 \%$ for the gangrene vignette, and $70 \%$ for the cancer vignette]. The type of vignette was significantly associated with the patient's indicated preference for family involvement $\left(\chi^{2}=9.9, \mathrm{P}=\right.$ $0.007)$, but not with the patient's preferred role $\left(\chi^{2}=4.7\right.$, $\mathrm{P}=0.32)$.
Multivariate analyses (ANCOVA) revealed that, after controlling for type of vignette, age and education were significantly associated with patient preference for decisionmaking (Partial $\mathrm{r}^{2}=0.03, \mathrm{P}=0.025$ for age; Partial $\mathrm{r}^{2}=$ $0.08, P=0.001$ for education). Older and less-educated patients were less likely to want to participate in decision making. Upon adjusting for type of vignette, education was also significantly associated with patients' preferences for family involvement (Partial $\mathrm{r}^{2}=0.04, \mathrm{P}=0.03$ ). Age and gender, however, were not associated with patients' preferences for family involvement.

The relationship between the patient's expressed role preference and desire for information is shown in Table 3. Patients who indicated a preference for a passive role were less likely to want information concerning treatment options, risks and benefits, and outcome probabilities, and were more likely to want to rely on the physician's recommendations, compared with those who preferred other roles. About $80 \%$ of the respondents would have sought the involvement of their spouses, and more than a half the involvement of their children.

\section{Who should have the final decision, in the face of disagreement?}

About $60 \%$ of the respondents for each vignette chose to respect the physician's recommendation when it conflicted with their own preference; while for 30\% their own preference remained paramount (Table 2). Very few respondents would give the family's opinion primary sway. Of the 16 respondents who would wish to decide alone or with their family members (Question E), five nevertheless answered that they would give their preference to a physician's opinion rather than their own, in the face of disagreement. Vignette had little influence on the choice of final decision-maker $(P=0.71)$, but preferred patient role showed a significant association (Table 4). Respondents who preferred an active role were more likely to prefer to make the final decision themselves, rather than have it made by the physician.

About $60 \%$ of the respondents for each vignette considered that no one should be held responsible for an adverse outcome of the treatment decided on, when it was appropriately performed, and less than $10 \%$ considered the physician should be held responsible (Table 2 ). The percentage who considered that the doctor should be held responsible did not differ for the three vignettes. More than $30 \%$ of those who preferred an active role considered that they themselves should be held responsible for the outcome of their treatment, while only about $10 \%$ of those who preferred other roles held this view. 


\section{Discussion}

Our primary question was whether Japanese patients are in fact passive as regards medical decision making. Current opinion is split over the level of patients' desire for self-determination and autonomy in Japan. Some argue that patient self-determination is not compatible with social mores in Japan, where collective decision-making is prevalent, and that patients are not accustomed to selfdetermination [21,22].

On the other hand, others are dissatisfied with the current physician paternalism and assert the patient's right to selfdetermination. A few years ago, a book entitled "At last I have caught a good doctor" became a much-discussed topic in the Japanese media [23]. The book describes the success story of a patient with a non-ruptured cerebral aneurysm, who refused the treatment of an arrogant and paternalistic surgeon, and found another surgeon whom she judged trustworthy. In order to find a good doctor, she made use of every available source of information, including electronic resources and cooperative friends, and interviewed several neurosurgeons. Her experiences aroused a sympathetic response in many readers who were tired of physician paternalism in Japan.

Japan has a long tradition of physician paternalism [24] as also seen in many other countries [25-27]. Ishiwata suggested that two deeply seated factors are major contributors to the paternalism of Japanese physician - an absence of the spirit of informed consent on the physician's side, and the practice of omakase (entrusting one's care to one's family and physician, on the assumption that they will make the decisions most beneficial or appropriate to oneself) on the patient's side [28]. As he put it, "Given the strong paternalism, patient passivity, and the exclusiveness among medical professionals in Japan, there is scarcely any opportunity for patients to participate in medical decision making." Others attribute physician paternalism to Japanese behavioral traits, religious beliefs, and their trust in nature. Morioka [29] and Kitao [30] concluded that the prevalent "mentality of individuals willing to entrust themselves to another person or to nature" and "the lack of self-consciousness regarding life and death," explain (at least partly) patient passivity as an aspect of the Japanese cultural tradition.

However the present study found that, compared with the United States, Canada, Israel and Russia $[7,20]$, there was no clear evidence that Japanese patients prefer more passive roles than do their counterparts in Western countries. A large majority of patients (75\% for the cancer vignette, and almost $90 \%$ for the non-cancer vignettes) held positive attitudes towards participation in medical decisionmaking, provided they received adequate information for the making of decisions.
Previous studies have suggested that patients do not want to make decisions on their own, although they do like to participate in determining the final decision. Benbassat, after reviewing published surveys of patients' preferences for participation in treatment decision making, concluded that, "Most respondents did not want an active role, but neither did they want to be entirely passive in the doctorpatient relationship [20]." Similarly, Strull et al. concluded that "patients prefer that decisions be made principally by their physicians, not themselves, although they very much want to be informed [9]." Katz has defined this apparently deceptive and incoherent attitude of patients as 'psychological autonomy', implying the motivation to become informed in order to exercise, or not, the right to self determination [16]. Even if armed with information, some patients may choose to express their autonomy by authorizing their physicians to make all the decisions, i.e. may decide not to decide. Although two-thirds of the subjects answered that they would prioritize their physician's judgment in the case of disagreement, the proportion of those who answered that "the doctor is responsible for the outcome of the selected treatment" was limited (ranged from $5 \%$ to $14 \%$ across the vignettes). A few subjects expressed that, "I, myself, am responsible for my treatment outcome, for I am who decides whether to follow my physician's advice." This statement also implies that the scope of "decision-making," understood by patients, carries a broad meaning. It not only denotes the act of deciding one's medical treatment but also connotes the act of judging whose opinion is the most beneficial for the patient.

In our study, the proportion of patients who preferred active roles ranged from $7 \%$ to $16 \%$ across the three vignettes. Nevertheless, a substantial number of patients considered their opinion to be most important in the final decision. Two thirds of respondents who preferred an active role and one third of those who preferred a collaborative role regarded themselves as the final decisionmakers when their opinions conflicted with those of their physicians. This finding suggests a conflict between the ethical principles of autonomy and beneficence: the paternalistic physician attempts to impose a medically beneficent management strategy on a patient who would, if adequately consulted, prefer to exercise some autonomy in the decisions made [31]. There is a possibility that one third of our study subjects fit this model.

Our second question concerned the factors that affect the patient's desire for decision participation. Our results indicated that, as in previous studies, older age and poor education were significantly associated with a patient preference for a passive role [9,32-36]. It is therefore suggested that higher socioeconomic status, as well as capacity to understand complicated medical information, are 
necessary to give patients the confidence to wish to be active participants in decision making.

The association between type of disease and patient preference for autonomy varied considerably with the question. Patients with the cancer vignette were more likely to express a preference for a passive role than those with the non-cancer vignettes $(25 \%$ vs. $12 \%)$, while the proportions of who preferred a collaborative role did not differ between the cancer $(68 \%)$ and non-cancer vignettes $(73 \%)$. There are several possible reasons for this difference. Firstly, the Japanese are not familiar with decision making in cancer treatment, since traditionally cancer patients have not been told the truth about their illness. Cancer is one of the most dreaded diseases in contemporary Japan, and until two decades ago, almost all Japanese, including physicians and patients, considered cancer disclosure out of the question [37]. A growing movement in support of disclosure over the past 15 years has raised public awareness of the issue, and data suggest that physicians in Japan have increasingly begun to disclose cancer diagnoses $[38,39]$, but the majority of physicians adhere to the policy of non-disclosure of a cancer diagnosis to patients $[40,41]$. In our study, patients' desire for information was consistently high in all vignettes, and was not significantly different in the cancer and the non-cancer vignettes. This finding raises the question of whether the Japanese still consider cancer an exceptional disease.

Secondly, in many cases the risks and benefits, and their trade-offs, for the various options for cancer treatment are too complicated for patients to fully understand, so that they are not confident about making difficult decisions. For example, patients' fear of regret for a bad personal decision could dispose them to have the decision taken out their hands. In our study, the only piece of information that the cancer vignette respondents were more likely wish for was the name of 'a famous specialist.' This suggests that in the face of cancer, patients may be more likely to wish to authorize their physician to make all decisions, to decide not to decide, and therefore wish to ensure the best chance of his/her decisions being correct [31].

An interesting finding was that even when the treatment options are quite simple and understandable, and so the decisions to be made were less difficult, many respondents still did not wish to make decisions on their own. For example, as many as 87 percent of the respondents with the pneumonia vignette, which asks for a very simple choice of one among three alternative treatments, and of one antipussive drug of two, chose to make their choice with the physician or to delegate the decision to the physician. This fact suggests that patients want physician's advice regardless of the severity of the disease or difficulty of the medical choice.
Our final study question concerned the role of the family in medical decision-making. Our study showed that although many patients wished to involve their families in decision making, very few would allow their family the final decision. This finding suggests that patients want their families to support their decision-making, rather than to provide a third opinion independent of both patient and physician. Thus, although we have attempted to analyze the clinical relationship in Japan as tripartite (i.e. patient, physician, and family), we have found that it is better described as bilateral, that is, 'physician' and 'patient plus family'. The character of the Japanese sense of 'self may partially account for the fuzzy boundaries between patient and family. Tamura and Lau have hypothesized that Japanese culture stresses the interconnectedness of persons, especially within the family, and thus the 'family self' is the 'basic, inner psychological organization' of the Japanese, and 'is intimately involved in the individual sense of "self"'. [42] Markus and Kitayama also defined the construal of the self in many non-Western cultures including Japan as "the interdependent construal." This term purports that culture insists on the fundamental connectedness of human beings resulting in the other being included within the boundaries of the self [43].

Conceivably, however, the alignment of patient and family is in part determined by the fact that in Japan patients may require the cooperation of their families for the fulfillment of their treatment decisions. Japanese inpatient hospital care is distinguished from that in Western countries by long hospitalization and continued care by the family. This practice does not only derive from Japanese culture and tradition $[37,44]$, but also from premature social infrastructures such as insufficient support by paramedical staff, and a lack of manpower and facilities in Japan's healthcare industry [2]. Although this situation is gradually changing, Japanese patients know that admission to a hospital may impose heavy burdens and workloads on their families. Therefore, obtaining the family's consent, their commitment to support and cooperation with hospital care, is an important consideration for Japanese patients.

Our study revealed that many Japanese patients consider that to make a decision on medical treatment is to reach an agreement between the patient, the physician, and the patient's family. Sometimes, patients' decisions may reflect a mixture of their own values and those of their families. In the discussion of treatment options, the patient's opinion has already been united with that of the family to create the "patient's preference for a treatment option". Usually, Japanese patients do not mind that their opinion is not independent of their families', nor do they 
imagine that their interests conflict with those of their families.

Certain limitations of our study need to be recognized. Firstly, although we used three different diseases (pneumonia, gangrene, and cancer) to manipulate severity of illness, we did not confirm whether or not subjects accurately shared with us the presumed severity of illness. However, in aims of presenting subjects with a better picture of illness severity, we provided information about the prognosis of each disease such as prospect of recovery and mortality: pneumonia was explained as a disease that would be cured with mere bed rest; gangrene was described with the probability of death following surgery being $1 \%$; and esophageal cancer was described with a 5year survival rate of $15-20 \%$. We believe that vignettes' severity of illness has a relatively high face-validity. Secondly, the study population was diabetic patients at a single clinic, so our results may not generalize to patients with other illnesses or in other settings. Diabetes is a disease that requires the patient's active participation in treatment to control blood glucose and to reduce the chances of developing sequellae, and most of our subjects were patients with relatively good compliance with treatment. Therefore, our study participants may have exhibited a greater desire for participation in treatment decisions than is general. We did not have a comparison group to identify the impact of diabetes on patients' desire for participation in decision making. However, several studies elsewhere have suggested that diabetic patients prefer less involvement in medical decision-making than do healthy adults or patients with other illness [45-47]. If these results also apply to the Japanese, it is unlikely that our study results underestimated patients' desire to participate in decision making in Japan. Secondly, since our study subjects were patients cared for by only two physicians, there may have been a selection bias caused by the particular physician-patient relationships. We did not investigate differences in the patients' desire for participation by physician, nor by duration of the illness. Further research will be necessary to determine whether our study results generalize to the whole population of Japan.

\section{Conclusions}

In summary, Japanese patients have positive attitudes towards participation in medical decision-making, provided they are fully informed of the nature of the disease, the treatment options, and the risks and benefits of the options. Physicians can encourage their participation by providing adequate medical information, as well as by supporting their decision-making, using their medical expertise and their knowledge of the patient's values. The family's involvement in medical decision making has important implications for Japanese patients, especially in cases of severe disease, since the Japanese system counts on continued patient care by the family. Physicians can increase patient satisfaction by accepting the role of helping their patients participate in decision-making and understanding their patients' wishes for family involvement, and so cultivating a democratic relationship in which decisions can be made cooperatively.

\section{Appendix \\ Questionnaire}

One of the following three vignettes was presented to each subject. The interviewer asked the subjects to select the number indicating the best management they feel about each statement if they actually had the medical condition.

\section{Pneumonia vignette}

You have had a productive cough for the past 5 days. You were well until a week ago, when you began to feel fatigued and feverish. The cough worsened, and now you are coughing up greenish brown sputum. The cough kept you up at last night.

There are three options for treatment: (1) Drug A is expensive, but has strong effects and requires minimal time for recovery; (2) Drug B is low-priced, but less effective and requires longer time for recovery than drug A; (3) Bed rest without medication can also cure the disease but takes at least 2 weeks. There are two options for cough suppressant: a very strong powder with more side effects, or a more mildly effective tablet with fewer side effects.

\section{Gangrene vignette}

After a penetrating foot injury, a few days ago your right foot began swelling and draining pus that smells bad. The painful foot keeps you up all night. You have had a high fever for the past 3 days.

One of the treatment options is to amputate your leg beneath the knee. Amputation is a relatively safety procedure, and the probability of death following surgery is $1 \%$. Another option is treatment with antibiotics. If the antibiotic is effective, amputation will not be necessary (in $30 \%$ of cases). However, if the antibiotic is not effective, the infection will be extended, and you must have your leg amputated above the knee. In that case, the chance of death after surgery is $10 \%$.

\section{Cancer vignette}

You have trouble swallowing. You were well until a few months ago, when you began to feel mid-chest discomfort. The discomfort gradually worsened, and now you cannot swallow solid foods such as rice and meat. You have lost $5 \mathrm{Kg}$.

You have been diagnosed with resectable esophageal cancer. The 5 year survival rate for this stage of the disease is 
$15-20 \%$. Surgery is the standard approach and can helpful for many reasons. In some cases, it can cure the cancer. It can also relieve the symptoms of the cancer. The death rate following surgery has decreased in the past 10 years and is well below $10 \%$. Surgery combined with chemoradiation can help to ease symptoms and extend or improve the quality of life. Chemo-radiation uses anticancer drugs along with radiation to kill the cancer. Anticancer drugs can have some side effects, depending on the type of drug, how much you take, and how long you take it. It is very common for patients to develop other health problems as a result of chemo-radiation, and the chance of dying from the therapy is as high as $15 \%$.

A. Do you want to consult a doctor immediately?

1. Yes

2. No

B. Given clinical diagnosis for the symptom, do you know what the treatment options are?

1. Not at all

2. A little

3. Pretty well

4. Know everything

C. The information is enormous and difficult to understand. However, your doctor is willing to explain anything you want to know. Regarding the treatment options, what kind of information do you require? $(1=$ Not at all; $2=$ Partially; $3=$ I want to know everything)

C-1. Treatment options: 1, 2, 3

C-2. Risks (adverse effects) and benefits of each treatment: $1,2,3$

C-3. How likely each of these risks and benefits are to happen (outcome probabilities): 1, 2, 3

C-4. The name of a famous specialist in this particular field: $1,2,3$

\section{C-5. Prognosis: 1, 2, 3}

C- 6 . Which treatment option the doctor thinks is the best (physician's recommendation): 1, 2, 3

D. Who should receive the information you selected above?

\section{Myself alone}

2. The doctor should tell me, and also let me decide which information my family should be given

3. The doctor should tell me and my family equally

4. The doctor should tell my family, and also let them decide which information I should be told

5. My family alone

E. Given all the information about risks and benefits of these possible treatments, who should decide the treatment?

1. Myself alone

2. Myself and my family alone

3. Myself and the doctor alone

4. The doctor, myself and my family equally

5. The doctor and my family alone

6. The doctor alone

7. Others

F. If the doctor's recommendation is different from your preference, whose opinion do you think is most important in deciding the treatment?

\section{My own}

2. My family's

3. The doctor's

4. Other

5. Don't know

G. If the treatment which had been chosen and performed appropriately did not work as expected, who shall be responsible for this result?

1. Myself

2. The doctor

3. Someone who made decision

4. No one is responsible for it 


\section{Other}

\section{Competing interests}

None declared.

\section{Authors' contributions}

MS and AA initiated the research, gathered data, conducted data analysis, and wrote the paper. TF participated in all processes and is guarantor for this paper. $\mathrm{MO}, \mathrm{NE}$ and TS participated in design and discussion. YI participated in revising the manuscript.

\section{Additional material}

\section{Additional File 1}

Table 1 - Demographic Characteristics of Respondents $(n=133)$. Table 2 - Questionnaire responses for the three vignette groups. Table 3 - Relationship of patient's preferred role to desire for various types of information. Table 4 - Association of patient's preferred role to choice of final decision-maker

Click here for file

[http://www.biomedcentral.com/content/supplementary/14712296-5-1-S1.doc

\section{Acknowledgements}

The authors would like to thank Dr Atshiko Satoh and Dr. Toshiko Kannaghi (Kyoto Preventative Medical Center) for their help in the collection of data. We also would like to thank Dr. Sara Carmel (Ben-Gurion University of Negev) for her comments on the study design.

\section{References}

I. Suzuki M, Konishi E, Soyano A, Ohta K. Ethical issues at the patients' end of life: Japanese nurses' perception. An international comparison of nurses' perception concerning ethical issues at the patients' end-of-life. in Final Report: International Comparison of Nurses' Awareness toward Ethical Issues in Terminal Care, Contract No: 096724 I 3. Sponsored by Ministry of Education 1999.

2. Kubo A, Nakagawa K, Miyamoto Y, Yana T, Itoh K, Kudoh S, Masuda N, Matsui K, Kusunoki Y, Takada M, Fukuoka M: Informed consent in the therapy of lung cancer patients. Nihon Gan Chiryo Gakkai Zasshi 1995, 30:664-679. In Japanese

3. Ishizaki T, Hisata M, Kai I, Ohi G, Yasumura Y, Hanawa M: Participation preference in therapeutic decision. Igaku No Ayumi 1993 166:585-586. In Japanese

4. Asai A: Should physicians tell patients the truth? West J Med 1995, 163:36-39.

5. Asai A, kobayashi Y, Fukuhara S: Preference of general public toward self-determination at the end of life. Igaku No Ayumi 1995, 173: 1031-1035. In Japanese

6. Toyokawa $\mathrm{H}$, Hirata $\mathrm{N}$ : Physician-patient relations: a time to establish new relationship. Nikkei Medical 1985, 14:28-37. In Japanese

7. Deber RB, Kraetschmer N, Irvine J: What role do patients wish to play in treatment decision-making? Arch Intern Med 1996, 156: | 4| 4- | 420 .

8. Ende J, Kazis L, Ash AB, Moskowitz MA: Measuring patients' desire for autonomy: decision making and information-seeking preference among medical patients. J Gen Intern Med 1989, 4:23-30.

9. Strull WM, Lo B, Charles G: Do patients want to participate in medical decision making? JAMA 1984, 252:2990-2994.

10. Ninchoji T, Sawai T, Tanaka S, Katagiri H, Abe Y, Shimoyama I: [Patient's comprehension of the medical informations pre- sented before the invasive procedures.]. Jpn Hosp 1993, 1 2:45-9. In Japanese

II. Kimura R: The right to be informed: An aspect of dignity. World Health Forum 1991, | 2:39|-92.

12. Kimura R: Death, dying, and advanced directives in Japan; sociocultural and legal points of view. Advanced directives and surrogate decision making in health care: United States, Germany and Japan Edited by: Sass HM, Veatch RM, Kimura R. Baltimore MD: Johns Hopkins University Press; 1998: 187-208.

13. Long SO: Family surrogacy and cancer disclosure: physicianfamily negotiation of an ethical dilemma in Japan. J Palliat Care 1999, I 5:31-42

14. Akabayashi A, Kai I, Takemura H, Okazaki H: Truth telling in the case of a pessimistic diagnosis in Japan. Lancet 1999, 354:1263.

15. Ruhnke GW, Wilson SR, Akamatsu T, Kinoue T, Takashima Y, Goldstein MK et al.: Ethical decision making and patient autonomy: a comparison of physicians and patients in Japan and the United States. Chest 2000, I I 8: I 172-82.

16. Katz J: The silent world of doctors a.d patient. Free Press, New York; 1984.

17. Veatch RM: Models for ethical medicine in a revolutionary age. Hastings Cent Rep 1972, 2:5-7.

18. Quill TE: Patnerships in patient care: a contractual approach Ann Intern Med 1983, 98:228-234.

19. Emanuel EJ, Emanuel LL: Four models of the physician-patient relationship. JAMA 1992, 267:222I-6.

20. Benbassat J, Pilpel D, Tidhar M: Patients' preferences for participation in clinical decision making: a review of published surveys. Behavioral Medicine 1998, 24:81-88.

21. Becker C: Ethical dilemma of Informed consent: Cost for avoiding a lawsuit, refusing to cooperate, and quasi-reliance. Igaku Tetsugaku Iryo Rinri 1999, I 7:85-94. In Japanese

22. Doi T: Amae no Ko-zo. (The anatomy of Dependence) Tokyo: Kobun-do: 1984. In Japanese

23. Shimoda H: Yatto Mei-I wo Tukamae-ta. (At last I have caught a good doctor: seventy-seven days before a brain surgery) Tokyo: Shincho-sha; 1999. In Japanese

24. Hayashi M, Hasui C, Kitamura F: Respecting autonomy in difficult medical settings: a questionnaire study in Japan. Ethics and Behavior 2000, 10:51-63.

25. Thomsen OO, Wulff HR, Martin A, Singer PA: What do gastroenterologists in Europe tell cancer patients? Lancet 1993 , 34I (8843):473-6.

26. Estape J, Palombo H, Hernandez E, Daniels M, Estape T, Grau JJ, Vinolas N, Mane JM: Cancer diagnosis disclosure in a Spanish hospital. Ann Oncol 1992, 3:45I-4.

27. Dalla-Vorgia P, Katsouyanni K, Garanis TN, Touloumi G, Drogari P, Koutselinis A: Attitudes of a Mediterranean population to the truth-telling issue.J Med Ethics 1992, 18:67-74.

28. Ishiwata $R$, Sakai A: The physician-patient relationship and medical ethics in Japan. Cambridge Quarterly of Healthcare Ethics 1994, 3:60-66.

29. Morioka M: Brain-Dead Person. Tokyo: Tokyo-Shoseki 1989:160

30. Kitao H: Communication in medical practice. In: The Parent Status of Bioethics Edited by: Tsukazaki S, Kamo N. Kyoto: Sekai Shisosha; 1989:179-80.

3I. Sutherland HJ, Llewellyn-Thomas HA, Lockwood GA, Tritchler DL, Till JE: Cancer patients: their desire for information and participation in treatment decisions. J R Soc Med 1989, 82:260-263.

32. Haug MR, Lavin B: Public challenge of physicians' authority. Med Care 1979, 17:844-858.

33. Cassileth BR, Zupkis RV, Sutton-Smith K, March V: Information and participation preferences among cancer patients. Ann Intern Med 1980, 92:832-836.

34. Degner LF, Sloan JA: Decision making during serious illness: What role do patient really want to play? J Clin Epidemiol 1992 , 45:94I-950.

35. Blanchard CG, Labrecque MS, Ruckdeschel JC, Blanchard EB: Information and decision-making preferences of hospitalized adult cancer patients. Soc Sci Med I988, 27: I I39-45.

36. Hack TF, Degner LF, Dyck DG: Relationship between preferences for decisional control and illness information among women with breast cancer. Soc Sci Med 1994, 39:279-289.

37. Ohnuki-Tierney E: Illness and culture in contemporary Japan. New York: Cambridge University Press; 1984 
38. Tanida $\mathrm{N}$ : Japanese attitudes towards truth disclosure in cancer. Scandinavian Journal of Social Medicine 1994, 22:50-57.

39. Elwyn TS, Ftters MD, Gorenflo DW, Tsuda T: Cancer disclosure in Japan: Historical comparisons, current practices. Soc Sci Med 1998, 46:115I-1163.

40. Hashimoto S: Taminaru kea ni taisuru sishiki ni kansuru kenkyu-kenkyu hokokusyo (Report on research concerning opinions toward terminal care) Ministry of Health and Welfare, Japan. .

4I. Elwyn TS, Fetters MD, Sasaki H, Tsuda T: Responsibility and cancer disclosure in Japan. Soc Sci Med 2002, 54:28I-293.

42. Tamura T, Lau A: Connectedness versus separateness: application of family therapy to Japanese families. Fam Process 1992, 3I:319-40.

43. Markus HR, Kitayama S: Culture and the self: implications for cognition, emotion, and motivation. Psychol Rev 1991, 98:224-253.

44. Yamamoto-Mitani N, Wallhagen MI: Pursuit of psychological wellbeing (ikigai) and the evolution of self-understanding in the context of caregiving in Japan. Cult Med Psychiatry 2002, 26(4):399-4I7.

45. Mansell D, Poses RM, Kazis L, Duefield CA: Clinical factors that influence patients' desire for participation in decisions about illness. Arch Intern Med 2000, 160:2991-6.

46. Anderson LA, DeVellis RF, Boyles BF, Feussner JR: Patients' perceptions of their clinical interactions: development of the multidimensional desire for control scales. Health Educ Res 1989, 4:383-97.

47. Pendleton L, House WC: Preferences for treatment approaches in medical care. College students versus diabetic outpatients. Med Care 1984, 22:644-6.

\section{Pre-publication history}

The pre-publication history for this paper can be accessed here:

http://www.biomedcentral.com/1471-2296/5/1/prepub

Publish with Biomed Central and every scientist can read your work free of charge

"BioMed Central will be the most significant development for disseminating the results of biomedical research in our lifetime. "

Sir Paul Nurse, Cancer Research UK

Your research papers will be:

- available free of charge to the entire biomedical community

- peer reviewed and published immediately upon acceptance

- cited in PubMed and archived on PubMed Central

- yours - you keep the copyright

Submit your manuscript here:

http://www.biomedcentral.com/info/publishing_adv.asp 